\title{
Characterization of Unlabeled Level Planar Trees
}

\author{
Alejandro Estrella-Balderrama ${ }^{\star}$, J. Joseph Fowler ${ }^{\star \star}$, \\ and Stephen G. Kobourov ${ }^{\star \star}$ \\ Department of Computer Science, University of Arizona \\ \{aestrell, jfowler, kobourov\}@cs.arizona.edu
}

\begin{abstract}
Consider a graph $G$ drawn in the plane so that each vertex lies on a distinct horizontal line $\ell_{j}=\{(x, j) \mid x \in \mathbb{R}\}$. The bijection $\phi$ that maps the set of $n$ vertices $V$ to a set of distinct horizontal lines $\ell_{j}$ forms a labeling of the vertices. Such a graph $G$ with the labeling $\phi$ is called an $n$-level graph and is said to be $n$-level planar if it can be drawn with straight-line edges and no crossings while keeping each vertex on its own level. In this paper, we consider the class of trees that are $n$-level planar regardless of their labeling. We call such trees unlabeled level planar (ULP). Our contributions are three-fold. First, we provide a complete characterization of ULP trees in terms of a pair of forbidden subtrees. Second, we show how to draw ULP trees in linear time. Third, we provide a linear time recognition algorithm for ULP trees.
\end{abstract}

\section{Introduction}

When drawing an $n$-vertex planar graph $G(V, E)$ in the $x y$-plane, a more restrictive form of planarity can be obtained by insisting on a predetermined $y$ coordinate for each vertex. In particular, suppose we have a set of $k$ equidistant horizontal lines or levels, namely $\ell_{j}=\{(x, j) \mid x \in \mathbb{R}\}$ for $j \in\{1,2, \ldots, k\}$ and each vertex is assigned to one of these $k$ levels. Call this level assignment $\phi$. The tuple $G(V, E, \phi)$ forms a $k$-level graph, and if $\phi$ is bijective so that each vertex is constrained to its own level, i.e., $k=n$, then $G(V, E, \phi)$ is an $n$-level graph. Further, suppose that when drawing $G$, each edge is a straight-line segment (or a continuous $y$-monotone polyline). If a planar drawing of $G$ can be obtained in spite of these restrictions, then $G$ is said to be level planar for level assignment $\phi$. If $G$ is an $n$-level graph that is level planar, then we say $G$ is $n$-level planar.

Some level assignments of $G$ do not allow for a level planar drawing. In fact, if $k<n$, then it is NP-hard [9] to determine whether there even exists a $k$-level assignment of $G$ in which $G$ is level planar. If $k=n$, in which $G$ is an $n$-level graph, such a level assignment gives a labeling of $V$ since each vertex in $V$ is uniquely numbered. A labeling of $V$ whose level assignment preserves the planarity of $G$ can be easily obtained from a plane drawing of $G$ and a perturbation

* Partially supported by CONACYT-México.

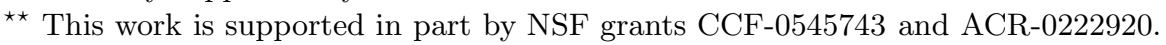

M. Kaufmann and D. Wagner (Eds.): GD 2006, LNCS 4372, pp. 367-379 2007.

(C) Springer-Verlag Berlin Heidelberg 2007 
of the vertices to ensure unique $x$-coordinates [2]. We call a $n$-level tree that is $n$-level planar for all possible labelings of its vertices an Unlabeled Level Planar (ULP) tree 1 . We characterize ULP trees in terms of a pair of forbidden subtrees and provide linear time recognition and drawing algorithms.

\subsection{Background and Related Work}

Visualizing hierarchical relationships has historically been a strong motivating factor in the study of the planarity of level graphs, i.e., graphs with a predetermined level assignment. Geometric simultaneous embedding has recently led to a new application of $n$-level graphs [2]. Determining which sets of graphs have geometric simultaneous embeddings has proven difficult. For instance, it is unknown whether a path and a tree can aways be simultaneously embedded. When simultaneously embedding an $n$-vertex path $P$ with another $n$-vertex graph $G$, one can relabel the vertices of $P$ sequentially 1 to $n$ from one endpoint to the other. The corresponding relabeling of the vertices of $G$ gives a natural level assignment $\phi$ for $G$. Eades et al. 4] have provided an $O(|V|)$ time algorithm for drawing any level planar graph with straight-line segments. Thus, if $G$ is $n$-level planar for $\phi$, it can easily be simultaneously embedded with $P$ since the path merely zig-zags in a $y$-monotone fashion from one level to the next. The ability to characterize $n$-level graphs gives additional insight into open problems in simultaneous embedding.

Jünger et al. [11] provide a linear time recognition algorithm for level planar graphs. This is based on the level planarity test given by Heath and Pemmaraju [78, which in turn extends the more restricted PQ-tree level planarity testing algorithm of hierarchies - level graphs of DAGs in which all edges are between adjacent levels and all the source vertices are on the uppermost levelgiven by Di Battista and Nardelli [3]. Hierarchies are characterized in terms of level non-planar (LNP) patterns in [3] as well. Jünger and Leipert [10] further provide a linear time level planar embedding algorithm that outputs a set of linear orderings in the $x$-direction for the vertices on each level. However, to obtain a straight-line planar drawing one needs to subsequently run a $O(|V|)$ algorithm given by Eades et al. 4] who demonstrate that every level planar embedding has a straight-line drawing. Healy et al. [6] use LNP patterns to provide a set of minimum level non-planar subgraph patterns that characterize level planar graphs. These subgraph patterns are somewhat analogous to Kuratowski's result that any minimal non-planar graph is either a subdivided $K_{5}$ or $K_{3,3}$ [12. It should be noted that these patterns are specific to a given level assignment and are not based solely on the underlying graph.

\subsection{Our Contribution}

Our contributions are three-fold. First, we provide a forbidden subdivision characterization for unlabeled level planar (ULP) trees in terms of two minimal ULP trees, $T_{1}$ and $T_{2}$; see Fig. 1 .

\footnotetext{
${ }^{1}$ A more appropriate name for these types of trees might be "unlabeled $n$-level planar
} trees" but for simplicity we call them unlabeled level planar or ULP trees. 


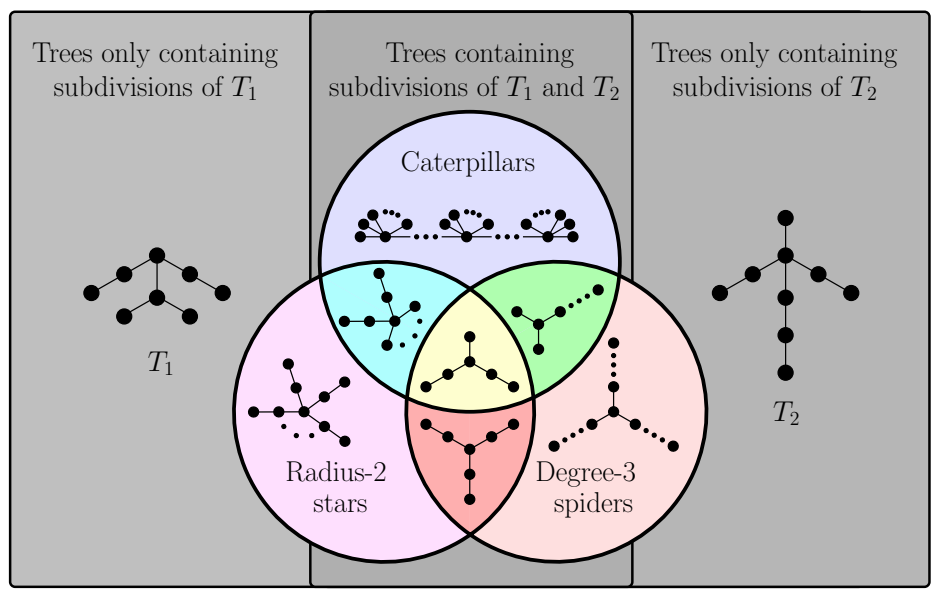

Fig. 1. A Venn diagram for the universe of trees characterized by the two forbidden subtrees $T_{1}$ and $T_{2}$. Graphs that do not contain either a subdivision of $T_{1}$ or $T_{2}$ are caterpillars, radius- 2 stars, and degree- 3 spiders.

Second, we characterize any tree without a subdivision of either $T_{1}$ or $T_{2}$ as either (i) a caterpillar, a tree in which the removal of all degree- 1 vertices yields a path, or (ii) a radius-2 star, a $K_{1, k}$ in which every edge is subdivided at most once, or (iii) a degree-3 spider, an arbitrary subdivision of $K_{1,3}$. We show that these three classes are $n$-level planar by virtue of an $O(|V|)$ time algorithm for constructing straight-line $n$-level planar drawings of ULP trees.

Third, if a tree is not a caterpillar, then it must contain a lobster (a graph in which the removal of all degree-1 vertices yields a caterpillar). Using minimal lobsters we show that trees that are not radius- 2 stars or degree- 3 spiders must contain subdivisions of $T_{1}$ or $T_{2}$, which completes the characterization. We also provide a $O(|V|)$ time algorithm for testing whether a tree falls into one of these three categories, thus yielding a linear time recognition algorithm for ULP trees.

\section{Preliminaries}

In this paper we try to use the established notation for level graphs whenever possible. The following definitions for levels graphs are predominantly taken from 136]. A $k$-level graph $G(V, E, \phi)$ on $n$ vertices is a DAG with a level assignment $\phi: V \rightarrow[1 . . k]$ such that the induced partial order is strict: $\phi(u)<\phi(v)$ for every $(u, v) \in E$. A $k$-level graph is a $k$-partite graph in which $\phi$ partitions $V$ into $k$ independent sets $V_{1}, V_{2}, \ldots, V_{k}$, which form the $k$ levels of $G$. A level- $j$ vertex $v$ is on the $j^{\text {th }}$ level $V_{j}$ of $G$ if $\phi(v)=j$ where $V_{j}=\phi^{-1}(j)$.

If $\phi$ is an injection, each level contains at most one vertex, i.e., $\left|V_{i}\right| \leq 1$ for $i \in[1 . . k]$, hence, $k \geq n$. W.l.o.g., we can assume in such instances that $k=n$ in which case $\phi$ is a bijection that forms a topological sort of the DAG $G(V, E)$. Unless noted otherwise, an $n$-level graph $G(V, E, \phi)$ is assumed to have a bijective 
level assignment $\phi$, i.e., $\phi: V \underset{\text { onto }}{\stackrel{1: 1}{\longrightarrow}}[1 . . n]$. Such a bijective level assignment is equivalent to a labeling of the vertices from 1 to $n$.

A level graph $G$ has a level drawing if there exists a drawing such that every vertex in $V_{j}$ is placed along the horizontal line $\ell_{j}=\{(x, j) \mid x \in \mathbb{R}\}$ and the edges are drawn as strictly $y$-monotone polylines. The order that the vertices of $V_{j}$ are placed along each $\ell_{j}$ in a level drawing induces a family of linear orders $\left(\leq_{j}\right)_{1 \leq j \leq k}$ along the $x$-direction, which form a linear embedding of $G$. A level drawing, and consequently its level embedding, is level planar if it can be drawn without edge crossings. A level graph $G$ is level planar if it admits a level planar embedding. The more restrictive definition of level drawings allowing only straight-line segments for edges is equivalent, as shown by Eades et al. [4. A planar graph $H$ is realized if it can be drawn with straight-line edges without crossings. Such a plane graph is a realization of $H$. A $n$-level graph $G(V, E, \phi)$ is $n$-level realized if it is realized such that each vertex $v$ lies on its level $\phi(v)$.

A chain of a $k$-level graph $G(V, E, \phi)$ is a nonrepeating sequence of vertices $v_{1}, v_{2}, \ldots, v_{t}$ of $V$ such that $t>1$ and either $\left(v_{i}, v_{i+1}\right) \in E$ or $\left(v_{i+1}, v_{i}\right) \in E$ for every $i \in\{1,2, \ldots, t-1\}$, i.e., a path in the underlying undirected graph of $G$. If $C$ is a chain, let LOWER $(C)$ and $\operatorname{UPPER}(C)$ denote the lowermost and uppermost vertices, respectively, with respect to $\phi$. Also let $\min (C)=\phi(\operatorname{LOWER}(C))$ and $\operatorname{MAx}(C)=\phi(\operatorname{UPPER}(C))$ be the minimum and maximum levels of $C$, thus, $\operatorname{MiN}(C) \leq \phi(v) \leq \operatorname{MAx}(C)$ for every $v$ of $C$.

For an $n$-level graph $G(V, E, \phi)$, let $<_{Y}$ denote the strict linear ordering given by the level assignment $\phi$, i.e., for every $u, v \in V$, we have $u<_{Y} v$ iff $\phi(u)<\phi(v)$. Let $<_{X}$ (and $\leq_{X}$ ) denote the strict (and weak) linear ordering induced by the $x$-coordinate of the placement of a level- $i$ vertex $u$ and a level- $j$ vertex $v$ along their respective horizontal lines $\ell_{i}$ and $\ell_{j}$ in the particular level drawing under consideration. Finally, for vertex subsets $U, W \subseteq V$, let $U<_{X} W$ (and $U<_{Y} W$ ) iff $u<_{X} w$ (and $u<_{Y} w$ ) for every $u \in U$ and $w \in W$. Often we will represent an edge $(u, v) \in E$ as $u-v$ and a chain of vertices, $v_{1}, v_{2}, \ldots, v_{t}$ for some $t>1$ as $v_{1}-v_{2}-\cdots-v_{t}$.

Finally, we recall a few standard graph theory definitions. In a graph $G(V, E)$, subdividing an edge $(u, v) \in E$ is the operation of replacing $(u, v)$ with the pair of edges $(u, w)$ and $(w, v)$ in $E$ by adding $w$ to $V$. A subdivision of $G$ is a graph obtained by performing a series of successive edge subdivisions of $G$.

\section{Characterization of Unlabeled Level Planar Trees}

First, we introduce the forbidden subdivisions $T_{1}$ and $T_{2}$ together with explicit level assignments in which the resulting graphs are level non-planar. Then we show how to compute a $n$-level realization for each of the three remaining types of trees - caterpillars, radius-2 stars and degree- 3 spiders - in linear time given a labeling of the vertices. Next, we show that if a tree does not contain a subdivision of $T_{1}$ or $T_{2}$, then it must fall into at least one of the three categories of unlabeled level planar trees. Finally, we give a simple $O(|V|)$ time recognition algorithm for ULP trees. Full details are included in the technical report $[5]$. 


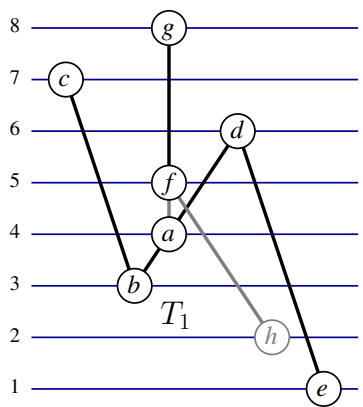

(a)

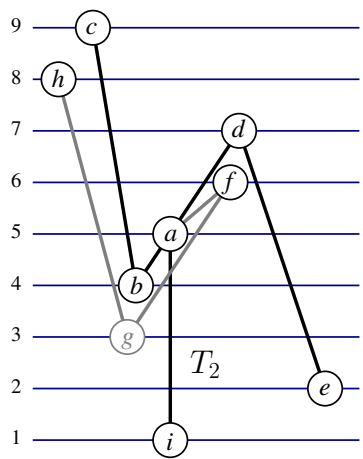

(b)

Fig. 2. Labelings that prevent $T_{1}$ and $T_{2}$ from being ULP

\subsection{Forbidden Trees}

Lemma 1. There exist labelings that prevent $T_{1}$ and $T_{2}$ from being level planar.

Proof. Fig. 2(a) gives one of 8 distinct labelings that satisfies the $y$-partial order $\{c, g\}>_{Y} d>_{Y} f>_{Y} a>_{Y} b>_{Y}\{e, h\}$ (or its dual in which the ordering is reversed). Each labeling gives a bijective $n$-level assignment in which $T_{1}$ does not have a $n$-level realization. This can be seen as follows: To prevent paths $a-b-c$ and $a-d-e$ from crossing, one path must go to the left and the other to the right. Assume w.l.o.g. $c-b<_{X} d-e$. This forces $c<_{X} f<_{X} d$ so that $a-f$ does not cross $b-c$, or $a-f-g$ does not cross $a-d-e$. However, then $f-h$ will cross either $a-d$ or $a-b-c$. This concludes the argument for $T_{1}$.

Next, consider $T_{2}$. Fig. 2(b) gives one of 8 distinct labelings that satisfies the $y$-partial order $\{c, h\}>_{Y} d>_{Y} f>_{Y} a>_{Y} b>_{Y} g>_{Y}\{e, i\}$ (or its dual in which the ordering is reversed). Each labeling gives a bijective $n$-level assignment in which $T_{2}$ does not have a $n$-level realization. This can be seen as follows: To prevent paths $a-b-c$ and $a-d-e$ from crossing, one path must go to the left and the other to the right. Assume again w.l.o.g. $c-b<_{X} d-e$. Then $a-i$ must be drawn below and to the right of $a-b$ and to the left of $d-e$, otherwise it will cross $b-c$ or $d-e$. To prevent the edge $a-f$ from crossing $a-b-c$ or $a-d-e, f$ must with be drawn (i) so that $a<_{X} f<_{X} e$ in which case $f-g-h$ will then cross $a-i$ or $d-e$, or (ii) so that $c<_{X} f<_{X} d$ in which case $f-g$ will cross $a-b-c, a-d$, or $a-i$. This completes the argument about $T_{2}$ and the overall claim.

Corollary 2. If a tree $T(V, E)$ contains a subdivision of $T_{1}$ or $T_{2}$, then it cannot be unlabeled level planar.

Proof. Assume that the tree $T$ contains a subdivision of $T_{1}\left(\right.$ or $\left.T_{2}\right)$. Let $T^{\prime}\left(V^{\prime}, E^{\prime}\right)$ be a subtree of $T$ that is a subdivision of $T_{1}$ (or $T_{2}$ ). Label the 8 (or 9 ) vertices of $V^{\prime}$ in the same order as shown in Fig. 2, Note that the values of the labels need to be adjusted in order to accommodate any intermediate vertices along a subdivided edge of $T_{1}$ (or $T_{2}$ ). Any extra vertex $w$ along a subdivided edge $(u, v)$ 


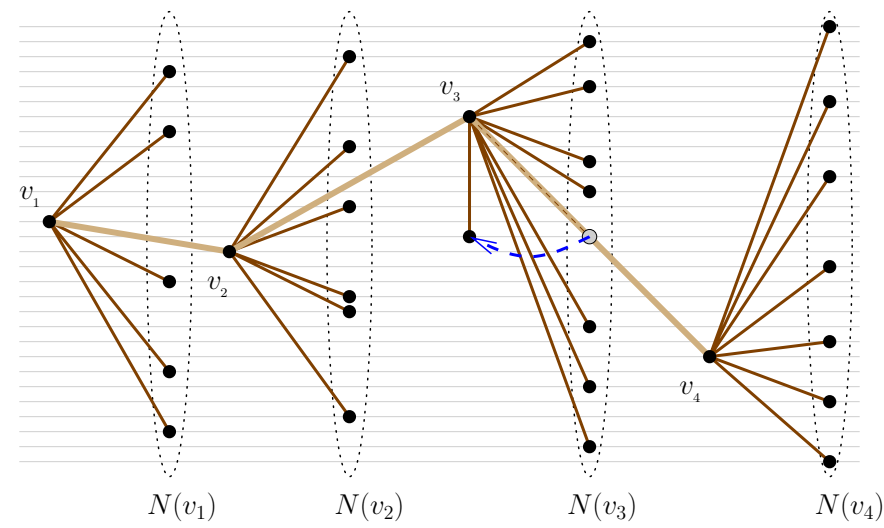

Fig. 3. A $n$-level realization of a 30 -level caterpillar on a $8 \times 30$ grid

can be assigned to a unique level that preserves the $y$-ordering $u<_{Y} w<_{Y} v$. This works since level planarity is defined in terms of $y$-monotone polylines. An edge drawn from level $\phi(u)$ to level $\phi(v)$ is allowed any number of bends so long as the edge proceeds in a $y$-monotone fashion. In particular, it can bend at $w$ on level $\phi(w)$, which is equivalent to subdividing the edge $u-v$ into $u-w-v$.

This gives a labeling of the vertices of $T^{\prime}$ using the labels $\left\{1,2, \ldots,\left|V^{\prime}\right|\right\}$ such that the 8 ( or 9 ) vertices corresponding to $T_{1}$ (or $T_{2}$ ) satisfy one of the $y$-partial orders from Lemma 1 Hence, the arguments used in Lemma 1 can be directly applied to this $y$-ordering. Thus, $T^{\prime}$ is not $n$-level planar, and as a consequence, neither is $T$, regardless of the labels for the remaining vertices.

\subsection{ULP Trees - Caterpillars, Radius-2 Stars, and Degree-3 Spiders}

The following three lemmas explicitly show all the trees that are unlabeled level planar and how to $n$-level realize them in linear time.

Lemma 3. (Brass et al. [2]) An n-vertex caterpillar $T(V, E)$ with an $m$-vertex spine can be $n$-level realized in $O(n)$ time on a $2 m \times n$ grid for any vertex labeling $\phi: V \underset{\text { onto }}{\stackrel{1: 1}{\longrightarrow}}\{1,2, \ldots, n\}$.

Proof. The following proof of the claim is a shorter version and is an improvement over the original proof in [3] that spans 3 pages. Note that the original claim had the slightly weaker result of using a $2 n \times n$ grid.

Let $T(V, E, \phi)$ be an $n$-level caterpillar with spine $S\left(V^{\prime}, E^{\prime}\right)$ such that $S$ is isomorphic to $P_{\left|V^{\prime}\right|}$. In particular, let the vertices of $V^{\prime}$ be labeled according to their relative distance from the end point $v_{1}$, and the edges $E^{\prime}=\left\{\left(v_{1}, v_{2}\right)\right.$, $\left.\left(v_{2}, v_{3}\right), \ldots,\left(v_{\left|V^{\prime}\right|-1}, v_{\left|V^{\prime}\right|}\right)\right\}$. Let the degree-1 leaves of $v$ be denoted by $N(v)=$ $\left\{u \mid(u, v) \in E\right.$ and $\left.(u, v) \notin E^{\prime}\right\}$ for each $v \in V^{\prime}$. Then for each $i \in\{1,2, \ldots, n\}$ place $v_{i} \in V^{\prime}$ at the coordinate $\left(2 i-1, \phi\left(v_{i}\right)\right)$ and place each $u \in N\left(v_{i}\right)$ at the coordinate $(2 i, \phi(u))$ unless $u$ would lie on the straight-line edge segment 


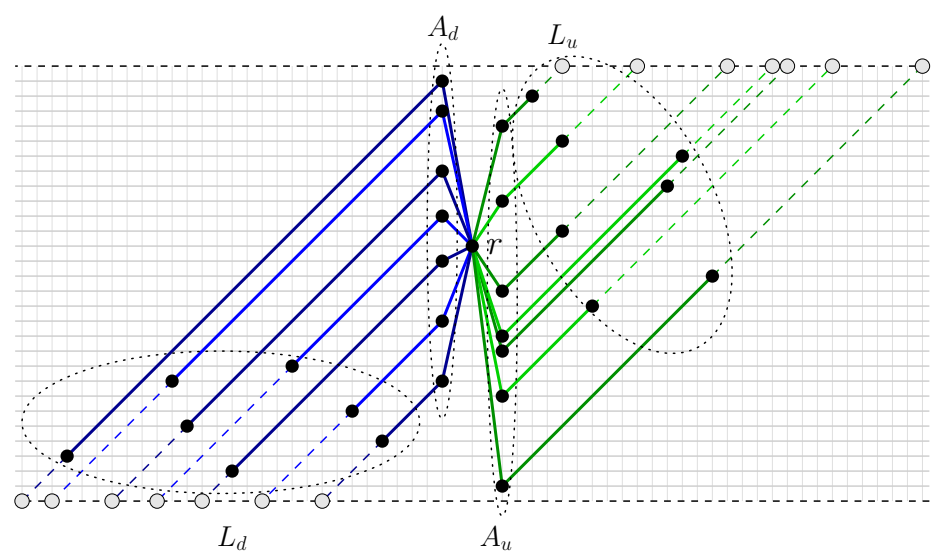

Fig. 4. A $n$-level realization of an 29 -level radius- 2 star on a $61 \times 29$ grid. The small gray circles are the intersection points of slope- 1 rays emanating from each vertex in $A_{d}$ and $A_{u}$ to the imagined 0-level and imagined $(n+1)$-level, respectively.

$v_{i}-v_{i+1}$ in which case place $u$ directly under $v_{i}$ at the coordinate $(2 i-1, \phi(u))$ instead. All this can be done in $O(n)$ time. This drawing is an $n$-level planar since $S$ is drawn in a strictly left to right fashion and each incident edge to the spine is either drawn either directly above or below the spine or immediately to its right. Clearly, this drawing uses only straight-line edge segments in which there are no crossings forming a $n$-level realization.

Lemma 4. An n-vertex radius-2 star $T(V, E)$ can be $n$-level realized in $O(n)$ time on $a(2 n+3) \times n$ grid for any vertex labeling $\phi: V \underset{\text { onto }}{\stackrel{1: 1}{\longrightarrow}}\{1,2, \ldots, n\}$.

Proof. Let $r$ be the root of an $n$-level radius- 2 star $T(V, E, \phi)$ located at the coordinate $(n+2, \phi(r))$. For every leaf $\ell$ that is at a distance of 1 from $\mathrm{r}$, place it at the coordinate $(n+1, \phi(\ell))$, which is one $x$-coordinate to the left of $r$. For each remaining leaf $\ell$, let $\operatorname{adj}(\ell)$ denote its adjacent vertex, and let $L \subseteq V$ denote this set of leaves at a distance 2 from $r$. Then $L_{d}=\{\ell \mid \ell \in L$ and $\phi(\operatorname{adj}(\ell))>\phi(\ell)\}$ and $L_{u}=\{\ell \mid \ell \in L$ and $\phi(\operatorname{adj}(\ell))<\phi(\ell)\}$ partition $L$ according to whether the adjacent vertex of the leaf is to be drawn above or below it, i.e., whether the incident edge goes down or up. Let $A_{d}=\left\{\operatorname{adj}(\ell) \mid \ell \in L_{d}\right\}$ and $A_{u}=\{\operatorname{adj}(\ell) \mid \ell \in$ $\left.L_{u}\right\}$ be the adjacent vertices of degree 2 to the leaf vertices of $T$.

Place each $u \in A_{d}$ at the coordinate $(n+1, \phi(u))$ immediately to the left of $r$, and each $u \in A_{u}$ at the coordinate $(n+3, \phi(u))$, immediately to the right of $r$. For each $\ell \in L_{d}$, place it at the grid point that corresponds to the intersection of the $\phi(\ell)$-level and the line segment connecting the points $(n+1, \phi(\operatorname{adj}(\ell))$, the coordinate of its adjacent vertex, and $(n-\phi(\operatorname{adj}(\ell))+1,0)$, the point that an emanating ray from $\operatorname{adj}(\ell)$ with a slope of 1 in the negative $x$-direction intersects an imagined 0-level; see Fig. 4. Since the ray has slope 1, this intersection will always be an integer grid point. In a similar fashion, place each $\ell \in L_{u}$ at the grid 
point that corresponds to the intersection of the $\phi(\ell)$-level and the line segment connecting the points $(n+3, \phi(\operatorname{adj}(\ell))$, the coordinate of its adjacent vertex, and $(n-\phi(\operatorname{adj}(\ell))+1, n+1)$, the point that an emanating ray from $\operatorname{adj}(\ell)$ with a slope of 1 in the positive $x$-direction intersects an imagined $(n+1)$-level. All this can be done in linear time since $O(1)$ time is spent locating each vertex.

This produces a $n$-level realization since every vertex that is adjacent to $r$ is either placed immediately to its right or left, and every other leaf is placed so that its incident edge has a slope of 1 , which prevents any edge from crossing.

Lemma 5. An n-vertex degree-3 spider $T(V, E)$ can be $n$-level realized in $O(n)$ time for any vertex labeling $\phi: V \underset{\text { onto }}{\stackrel{1: 1}{1}}\{1,2, \ldots, n\}$.

Proof. The proof is in three parts. First, we show how to reduce an arbitrary degree-3 spider to one in which all the legs zig-zag between successively lower and higher levels. Second, we skip ahead and show how to place the vertices of the original spider when processing an edge of the reduced spider. Finally, we show an $O(n)$ time algorithm for greedily drawing the reduced degree-3 spider.

Part 1: Let $r$ be the root vertex of an $n$-level degree-3 spider $T(V, E, \phi)$. Let $X, Y$, and $Z$ be the three subtrees of $r$, each of which forms a chain. Call $T^{\prime}\left(V^{\prime}, E^{\prime}, \phi^{\prime}\right)$ a strictly expanding degree-3 spider if the level assignment $\phi^{\prime}$ on the vertices $r, v_{2}, \ldots, v_{|C|}$ of each chain $C$ of $T^{\prime}$ obeys the following two properties:

and

$$
\phi\left(v_{i-1}\right)<\phi\left(v_{i}\right)>\phi\left(v_{i+1}\right) \text { or } \phi\left(v_{i-1}\right)>\phi\left(v_{i}\right)<\phi\left(v_{i+1}\right),
$$

$$
\begin{aligned}
& {\left[\phi\left(v_{i-1}\right)<\phi\left(v_{i}\right) \Rightarrow \phi\left(v_{i-1}\right)>\phi\left(v_{i+1}\right)\right] \text { and }} \\
& {\left[\phi\left(v_{i-1}\right)>\phi\left(v_{i}\right) \Rightarrow \phi\left(v_{i-1}\right)<\phi\left(v_{i+1}\right)\right]}
\end{aligned}
$$

for $1<i<|C|$. We call a chain that satisfies property (1) a zig-zagging chain since it cannot have any monotonically increasing or decreasing sequences of

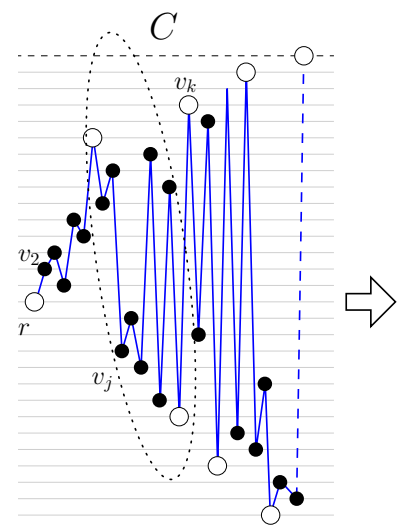

(a)

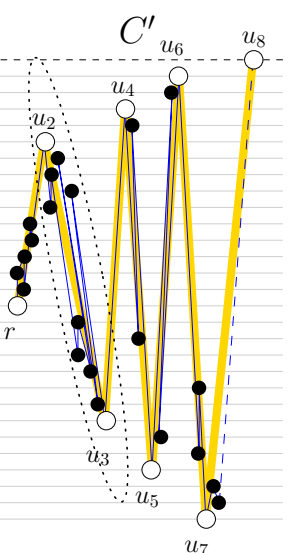

(b)

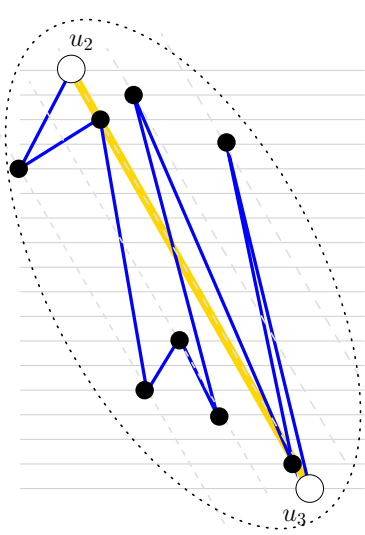

(c)

Fig. 5. An example of a chain $C$ of (a) in which the strictly expanding zig-zagging subchain $C^{\prime}$ of white vertices is extracted to give (b). Then (c) shows an example $n$-level realization of the intermediate edges and vertices for the second edge of (b). 


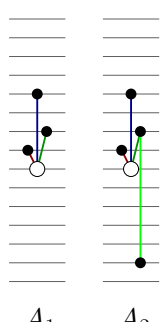

$A_{1}$

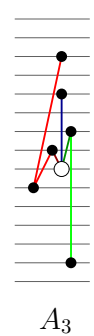

$A_{3}$

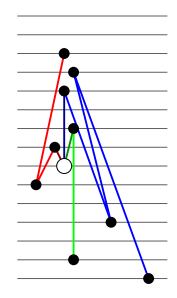

$A_{4}$

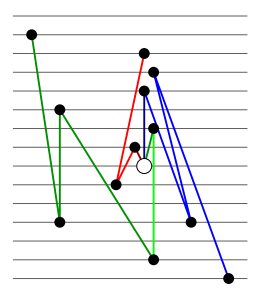

$A_{5}$

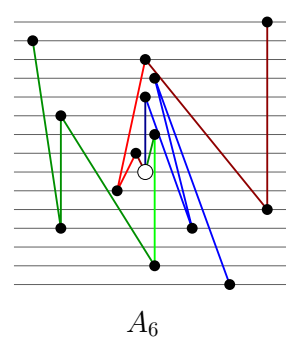

$A_{6}$

Fig. 6. Six iterations of the greedy strategy to $n$-level realize a degree- 3 spider

vertices. A zig-zagging chain that also satisfies property (2) is strictly expanding as the next level reached by the chain is either greater than any previous level or less than any previous level.

A zig-zagging chain $C$ can be made strictly expanding by keeping track of the minimum and maximum levels encountered by the chain so far. Assume w.l.o.g. that $\phi(r)<\phi\left(v_{2}\right)$, i.e., the chain $C$ begins by going upwards. We extract from $C$, the strictly expanding subchain $C^{\prime}$, which we will label its vertices as $r-u_{2}-u_{3}-\cdots-u_{C^{\prime}}$, by first prepending $r$ to $C^{\prime}$. Then we set $\min _{C}=\phi(r)$ and find the first vertex $v_{j}$ along $C$ such that $\phi\left(v_{j}\right)<\min _{C}$. Next we append $u_{2}=\operatorname{UpPeR}\left(r-v_{2}-v_{3}-\cdots-v_{j-1}\right)$ to $C^{\prime}$, and set $\max _{C}=\phi\left(u_{2}\right)$. Then we look for the next vertex $v_{k}$ along $C$ such that $\phi\left(v_{k}\right)>\max _{C}$. Afterward, we append $u_{3}=\operatorname{LOWER}\left(v_{j}-v_{j+1}-\cdots-v_{k-1}\right)$ to $C^{\prime}$, and set $\min _{C}=\phi\left(u_{3}\right)$, and repeat this process until all the vertices of $C$ are exhausted. If the last vertex encountered is not greater than $\min _{C}$ or less than $\max _{C}$, then we add an extra vertex to the end of $C^{\prime}$ satisfying this condition; see Fig. 5 for an illustration.

Part 2: For each vertex $u_{i}$ in $C^{\prime}$, we keep a linked list of the subpath $P=u_{i}-w_{i_{1}}-w_{i_{2}}-\cdots-w_{i_{|P|-2}}-u_{i+1}$ of $C$ that was replaced by the edge $u_{i}-u_{i+1}$ where $\operatorname{Min}(P)=\phi\left(u_{i}\right)$ and $\operatorname{MAx}(P)=\phi\left(u_{i+1}\right)\left(\right.$ or $\operatorname{MAx}(P)=\phi\left(u_{i}\right)$ and $\left.\operatorname{MIN}(P)=\phi\left(u_{i+1}\right)\right)$. This linked list will be used to place edges of $T$ as we process edges from $T^{\prime}$. Fig. 5( (c) visually illustrates how this might be done. Here, any particular subpath $P$ of $C$ for a given edge of $C^{\prime}$ can be drawn arbitrary close to $C$. We omit the details of this particular point, noting only that the intuitive idea of compressing the zig-zagging chain allows us to greedily draw the edges of $T$ without crossings for each edge of $T^{\prime}$ that is processed. We finish this section of the proof by observing that both the extraction of $C^{\prime}$ from $C$ and the ability to draw the edges of $T$ once we have processed the edges of $T^{\prime}$ can be done in linear time.

Part 3: Now that we have our degree-3 spider in the proper form, we can apply a simple greedy algorithm that can be used to give a $n$-level realization of $T^{\prime}$. We complete the proof of the lemma by giving the details regarding this linear time algorithm.

Let the vertices of chain $X$ be denoted by $x_{0}-x_{1}-\cdots-x_{|X|-1}$ in which $x_{0}=r$ and $\left(x_{i}, x_{i+1}\right) \in E$ for $0 \leq i<|X|-2$. Similarly, let the vertices of $Y$ and $Z$ be 
$y_{0}-y_{1}-\cdots-y_{|Y|-1}$ and $z_{0}-z_{1}-\cdots-z_{|Z|-1}$. Finally, let $A_{1}=\left\{x_{1}, y_{1}, z_{1}\right\}$ be the first vertices along each chain immediately following $r$.

There exist two possibilities for the strictly expanding degree-3 spider $T^{\prime}$ : Either (i) $A_{1}>_{Y} r$ (or $A_{1}<_{Y} r$ ) or (ii) $\operatorname{MIN}\left(A_{1}\right)<_{Y} r<_{Y} \operatorname{MAX}\left(A_{1}\right)$. We show how to draw $T$ for case (i) assuming that $A_{1}>_{Y} r$. The other case is similar. We start the first iteration by drawing $A_{1}$ so that the vertex of maximum index with respect to $\phi^{\prime}$ lies between the other two vertices of $A_{1}$ along the $x$-coordinate.

At any one point in this greedy strategy, we maintain the invariant that the last vertex along a chain that we placed either lies above or below any of the other vertices that have been drawn so far. Property (2) allows us to do this. If we encounter the end of a chain in which this invariant does not hold for its last vertex, then we can easily draw the remaining two chains without crossings. We do this by drawing one of the two chains monotonically to the right until we reach its end, and do the same for the other chain monotonically to the left.

For iteration $i>1$, we arbitrarily pick one of the chains whose most recently placed vertex is neither the maximum nor the minimum vertex drawn so far. We greedily extend the chain either to the right or left until we reach a vertex whose level assignment is either above or below all the ones drawn so far. This enlarges the set of processed vertices from $A_{i-1}$ to $A_{i}$. Note that we can always extend a chain $C$ to the right or left. This follows from the fact that during the previous iteration, before the vertices of some other chain $C^{\prime}$ were processed, the last vertex $v$ of $C$ was either minimum or maximum; see Fig. 6 for an example.

Since we can always greedily place a vertex without introducing a crossing, this strategy succeeds in producing a $n$-level realization of $T$ in $O(n)$ time (constant time per vertex), which shows that $T$ is indeed ULP. Simple geometry can be used to construct such an drawing using only straight-line edge segments for $T^{\prime}$, which can be used to produce a plane drawing of $T$ as detailed above.

Now that we have shown which trees are ULP, we need to show that our characterization is complete. First, we show that $T_{1}$ and $T_{2}$ are minimal unlabeled level non-planar trees with the following lemma.

\section{Lemma 6. Removing any edge from $T_{1}$ or $T_{2}$ yields a forest of ULP trees.}

Proof. If removing an edge from $T_{1}$ decreases the degree of one of the two degree-3 vertices, call them $x$ and $y$, then the resulting graph is a forest consisting of a degree-3 spider and a possible lone edge; see Fig. 7(a). Removing the edge $x-y$ yields two paths. The only possibility (up to isomorphism) in removing an edge without affecting the degree of $x$ and $y$, yields a caterpillar with a spine of length 5 . Moving onto $T_{2}$, if its vertex $z$ of degree 4 maintains its degree after the edge removal, then the resulting graph must be a forest consisting of either a caterpillar, if the removed edge was incident to a leaf vertex at a distance 2 from $z$, or a radius- 2 star and a possible lone edge, otherwise. On the other hand, if the degree of $z$ decreases to 3 , then the resulting graph is a degree- 3 spider and, possibly, a path; see Fig. [7(c).

The next theorem completes the characterization of ULP trees. 


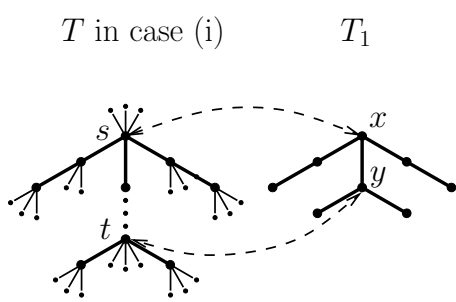

(a)

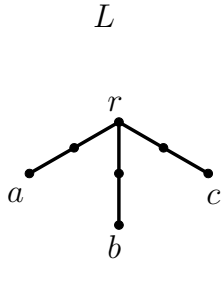

(b)

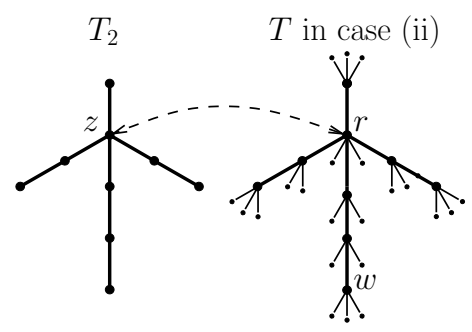

(c)

Fig. 7. Finding copies of $T_{1}$ and $T_{2}$

Theorem 7. Every tree either contains a subdivision of $T_{1}$ or $T_{2}$ in which case it is not ULP, or it is a caterpillar, a radius-2 star, or a degree-3 spider in which case it is ULP. Hence, $T_{1}$ and $T_{2}$ give a minimal forbidden subtree characterization of ULP trees.

Proof. First, we argue that neither $T_{1}$ nor $T_{2}$ is a caterpillar, a radius- 2 star, or a degree-3 spider to show that if a tree $T$ contains a subdivision of $T_{1}$ or $T_{2}$, it cannot be one of those three. Clearly, both $T_{1}$ and $T_{2}$ are lobsters and not caterpillars. The two vertices of degree 3 prevent $T_{1}$ being a radius- 2 star or a degree- 3 spider. Since $T_{2}$ has radius 3 and a vertex of degree 4 , it cannot be a radius-2 star or a degree-3 spider either.

Now we show that caterpillars, radius- 2 stars, and degree- 3 spiders are the only types of ULP trees. We do this by showing that any tree that does not fit into at least one of these categories must contain either a subdivision of $T_{1}$ or $T_{2}$. Then by Corollary 2 any tree $T$ that contains a subdivision of $T_{1}$ or $T_{2}$ cannot be ULP. By Lemma 6, $T_{1}$ and $T_{2}$ are minimal.

Assume that $T(V, E)$ is a tree that is not a caterpillar, radius-2 star, or degree-3 spider. Since $T$ is not a caterpillar, it must contain a minimal lobster $L$, i.e., the unique tree that cannot have any more edges removed without becoming a caterpillar (and possibly a lone edge); see Fig. 7(b). It has one vertex $r$ of degree 3 and three leaf vertices $a, b, c$ at a distance 2 from $r$, which is the minimal requirement for a tree to be a lobster. Any other lobster can have its edges trimmed away until $L$ is all that remains, which is what makes $L$ minimal.

Since $T$ is not a degree-3 spider, there are two cases to consider: either (i) $T$ has two vertices $s$ and $t$ of degree at least 3 or (ii) $T$ has one vertex of degree $k$ greater than 3 .

Assuming case (i) holds, we show how to find a subdivision of $T_{1}$ in $T$. Let $x$ and $y$ be the two vertices of degree 3 in $T_{1}$ where $x$ is the one without an adjacent leaf vertex; see Fig. 7(a). At least one of the two vertices $s$ and $t$ of degree at least 3 in $T$ must correspond to the root vertex $r$ in the subtree $L$ that forms the minimal lobster in $T$. Assume w.l.o.g. this vertex is $s$. Then we map $s$ in $T$ to vertex $x$ in $T_{1}$, and the other vertex of degree at least $3, t$ in $T$ to vertex $y$ in $T_{1}$. Since $t$ has degree at least 3 , there exists two neighbors of $t$ not along the path from $s$ to $t$, which we can map to the two corresponding leaf vertices 
in $T_{1}$ that are adjacent to $y$. Only one of the three leaf vertices $a, b, c$ of $L$ in $T$ can be contained in the subtree of $s$ containing $t$. Suppose w.l.o.g. it is $a$. Then the other two vertices $b$ and $c$ in $T$ can be mapped to the two remaining leaf vertices in $T_{1}$. This completes the mapping of vertices of $T_{1}$.

Next we consider case (ii) in which we show how to find the subtree $T_{2}$ in $T$. The one vertex of degree $k$ greater than 3 must be the corresponding vertex $r$ of $L$ in $T$; see Fig. 7(c). Otherwise, if there were separate vertices of degree greater than 3 , case (i) would apply. Let $r$ be mapped to the degree-4 vertex $z$ of $T_{2}$. Since $T$ is not a radius- 2 star, there exists a vertex $w$ at a distance 3 from $r$, which can be mapped to the leaf vertex in $T_{2}$ at a distance 3 from $z$. Only one of the three vertices $a, b, c$ of $L$ in $T$ can be along the path from $r$ to $w$. Suppose w.l.o.g. it is $a$. The other two vertices $b$ and $c$ in $T$ can be mapped to the other two leaf vertices in $T_{2}$. The remaining leaf vertex of $T_{2}$ that is directly adjacent to $z$ can be mapped to the endpoint of the fourth edge incident to $r$ in $T$ since it has degree greater than 3. This completes the mapping of vertices of $T_{2}$.

\subsection{Linear Time Recognition of ULP Trees}

First, we need a few simple observations regarding the degree sequences of caterpillars, radius-2 stars, and degree-3 spiders, which we state as lemmas whose proofs we omit in this abstract.

Lemma 8. If a tree $T$ has a degree sequence of the form $2, \ldots, 2,1,1$ or 1,1 , i.e., a path, after the removal of all degree-1 vertices, then $T$ must be a caterpillar.

Lemma 9. If a tree $T$ has a degree sequence of the form $k, 2, \ldots, 2,1,1, \ldots 1$ for some $k>2$, i.e., $T$ is an arbitrarily subdivided $K_{1, k}$, and after the removal of all degree-1 vertices, the degree sequence then becomes $\ell, 1, \ldots, 1$ for some $\ell \leq k$, i.e., $T$ becomes a $K_{1, \ell}$, then $T$ must be a radius -2 star.

Lemma 10. If a tree $T$ has a degree sequence of the form $3,2, \ldots, 2,1,1, \ldots 1$, i.e., $T$ has maximum degree of 3 with only one vertex of degree 3 , then $T$ must be a degree-3 spider.

Theorem 7 together with the above Lemmas, lead to a simple linear time recognition algorithm for ULP trees summarized in the following corollary:

Corollary 11. The class of ULP trees can be recognized in linear time. That is, given an arbitrary $n$-vertex tree $T$, one can decide in $O(n)$ time whether or not it is always possible to $n$-level realize $T$ for any possible labeling.

\section{Conclusion and Future Work}

We described a complete characterization of unlabeled level planar trees. We provided a linear time algorithm to $n$-level realize the three classes of ULP trees which can also be used for simultaneously embedding a ULP tree $T$ with any 
path $P$. Finally, we provided a linear time recognition algorithm for ULP trees. What is missing from the recognition algorithm is a certificate of unlabeled level non-planarity, i.e., the 8 (or 9) vertices corresponding $T_{1}$ (or $T_{2}$ ) if they exist.

Another future task is to provide a forbidden subgraph characterization for general unlabeled level planar graphs as we have done for ULP trees.

\section{References}

1. C. Bachmaier, F. J. Brandenburg, and M. Forster. Radial level planarity testing and embedding in linear time. J. Graph Algorithms Appl., 9(1):53-97, 2005.

2. P. Brass, E. Cenek, C. A. Duncan, A. Efrat, C. Erten, D. Ismailescu, S. G. Kobourov, A. Lubiw, and J. S. B. Mitchell. On simultaneous graph embedding. In 8th Workshop on Algorithms and Data Structures, pages 243-255, 2003.

3. G. Di Battista and E. Nardelli. Hierarchies and planarity theory. IEEE Trans. Systems Man Cybernet., 18(6):1035-1046 (1989), 1988.

4. P. Eades, Q. Feng, X. Lin, and H. Nagamochi. Straight-line drawing algorithms for hierarchical graphs and clustered graphs. Algorithmica, 44(1):1-32, 2006.

5. A. Estrella-Balderrama, J. J. Fowler, and S. G. Kobourov. Characterization of ulabeled planar trees. Technical Report TR06-03, University of Arizona, 2006.

6. P. Healy, A. Kuusik, and S. Leipert. A characterization of level planar graphs. Discrete Math., 280(1-3):51-63, 2004.

7. L. S. Heath and S. V. Pemmaraju. Recognizing leveled-planar dags in linear time. In 4th Symposium on Graph Drawing (GD), pages 300-311. 1996.

8. L. S. Heath and S. V. Pemmaraju. Stack and queue layouts of directed acyclic graphs. II. SIAM J. Comput., 28(5):1588-1626, 1999.

9. L. S. Heath and A. L. Rosenberg. Laying out graphs using queues. SIAM J. Comput., 21(5):927-958, 1992.

10. M. Jünger and S. Leipert. Level planar embedding in linear time. J. Graph Algorithms Appl., 6:no. 1, 67-113, 2002.

11. M. Jünger, S. Leipert, and P. Mutzel. Level planarity testing in linear time. In 6th Symposium on Graph Drawing (GD), pages 224-237. 1998.

12. C. Kuratowski. Sur les problèmes des courbes gauches en Topologie. Fundamenta Mathematicae, 15:271-283, 1930. 